\title{
Study of incidence, clinical profile and risk factors of neonatal hypoglycemia in a tertiary care hospital
}

\author{
Manjunatha Babu R ${ }^{1}$, Joy L. P. D'Souza², Susheela $C^{3}$ \\ ${ }^{1}$ Dr. Manjunatha Babu R, Associate Professor, ${ }^{2}$ Dr. Joy Liston Pratap D’ Souza, Associate Professor, ${ }^{3}$ Dr. Susheela C, \\ Professor; all authors are affiliated with Department of Pediatrics, Vydehi Institute Medical Sciences and Research \\ Centre, Bengaluru, Karnataka, India.
}

Address for Correspondence: Dr. Manjunatha Babu R, Associate Professor, Department of Paediatrics, Vydehi Institute of Medical Sciences and Research Centre, White field, Bengaluru. Email id: manjunath.babu9@gmail.com

\begin{abstract}
Background: The reported incidence of hypoglycemia varies with its definition. The clinical manifestations of hypoglycemia are nonspecific and similar to those of many disorders in newborn infants. Persistent hypoglycemia is more likely to be associated with possible neurologic sequelae. However, its definition, clinical significance, and management remain controversial. Methods: This prospective observational study was done over a period of 2 years. Babies admitted to Neonatal intensive care unit with whole blood sugar levels $<40 \mathrm{mg} / \mathrm{dl}$ and fulfilling inclusion criteria were taken up for the study. These babies were subjected to detailed history taking, thorough clinical examination and investigations. The babies were observed for signs and symptoms of hypoglycemia. Results: Neonatal hypoglycemia constituted about $8.26 \%$. Majority of hypoglycemic babies were preterm babies. Asymptomatic hypoglycemia was predominantly noticed in preterm babies $(65.21 \%)$. Term babies $(58.82 \%)$ showed more symptoms with hypoglycemia than preterm babies. The major clinical manifestations are jitteriness, lethargy, convulsions and apneic spells. PIH and prematurity are most associated risk factors. Significantly low Sugar levels (p value <0.005) were noticed in symptomatic hypoglycemic babies. Conclusion: Hypoglycemia in neonates can have variable presentations indicating the need for detailed and thorough examination for evidence of hypoglycemia. Identification of risk factors of hypoglycemia and proper monitoring blood glucose levels should be done to plan early treatment and prevent neurological damage.
\end{abstract}

Key words: Blood glucose levels, Hypoglycemia, Newborn, Risk Factors.

\section{Introduction}

Neonatal hypoglycemia has been recognized for many years, although with time there have been wide swings of opinion regarding the definition of the condition, its clinical significance and its optimal management. For example, in the era when routine postnatal management involved the withholding of feeds from healthy infants for up to 24 hours, even longer in sick or smaller babies, many were found to have low blood glucose concentrations and this became accepted as a normal finding [1]. The reported incidence of hypoglycemia varies with its definition, but it has been estimated to occur in approximately $16 \%$ of large-for-gestationalage (LGA) infants and $15 \%$ of small-for-gestational-age (SGA) babies [2]. Blood-glucose levels are frequently lower in newborn babies than in older children or

Manuscript received: $24^{\text {th }}$ September 2016

Reviewed: $4^{\text {th }}$ October 2016

Author Corrected; $15^{\mathrm{h}}$ October 2016

Accepted for Publication: $27^{\text {th }}$ October 2016 adults, but confirming a diagnosis of clinically significant hypoglycemia requires that one interpret the blood glucose level within the clinical context [2]. The clinical manifestations of hypoglycemia are nonspecific and similar to those of many disorders in newborn infants. The clinical signs and symptoms should improve with correction of low glucose concentration [3]. Most cases of neonatal hypoglycemia are transient, respond readily to treatment and are associated with an excellent prognosis. Persistent hypoglycemia is more likely to be associated with abnormal endocrine conditions and possible neurologic sequel [2]. Hypoglycemia is one of the most common metabolic problems seen in both the newborn nursery and neonatal intensive care unit (NICU). However its definition, clinical significance and management remain controversial. Studying the incidence may help to plan the services, identifying the clinical features and risk 
factors associated with neonatal hypoglycemia, may help in preventing neurological damage.

\section{Material and Methods}

This is a case series study done at a tertiary care hospital over a period of 2 years. Babies admitted to Neonatal intensive care unit with whole blood sugar levels $<40 \mathrm{mg} / \mathrm{dl}$ were taken up for the study. A written informed consent was taken from either of the parents of the babies. Babies with Congenital malformations, Neonatal cord injuries, inborn errors of metabolism, maternal history of oral hypoglycemic agents, beta sympathomimetics and maternal glucose infusions during delivery were excluded from the study. All babies admitted to Neonatal intensive care unit were subjected to Random Blood Glucose estimation initially by strip method using a Glucometer and in babies who show blood sugar levels $<40 \mathrm{mg} / \mathrm{dl}$, a second blood sample was sent to laboratory immediately, for estimation of whole blood sugar levels by oxidase method using autoanalyser. Babies with whole blood sugar levels less than $40 \mathrm{mg} / \mathrm{dl}$ in both the samples were taken up for study. These babies were subjected to detailed history taking, thorough clinical examination and investigations. After taking detailed history, various associated maternal risk factors like gestational diabetes mellitus, pregnancy induced hypertension antepartum hemorrhage, twin pregnancy and neonatal risk factors like prematurity (Assessed by Ballard's score), Intrauterine growth restriction IUGR (Assessed by growth charts), sepsis (Septic screen and blood culture and sensitivity), birth asphyxia (APGAR score $<7$ ) were recorded. The babies were managed and treated as per standard protocol. The babies were observed for signs and symptoms of hypoglycemia. The data was collected, analyzed and compared after calculating percentages, mean values with standard deviations accordingly.

\section{Results}

A Total of 484 cases were admitted to NICU during the study period and 40 cases had hypoglycemia. Neonatal

Table-1: Clinical Profile.

\begin{tabular}{|c|c|c|}
\hline Characters & Number of cases $(n=40)$ & Percentage $(\%)$ \\
\hline \multicolumn{3}{|l|}{ Gestational age } \\
\hline Term & 23 & 57.5 \\
\hline Preterm & 17 & 42.5 \\
\hline \multicolumn{3}{|l|}{ Sex } \\
\hline Male & 23 & 57.5 \\
\hline Female & 17 & 42.5 \\
\hline \multicolumn{3}{|l|}{ Nature of Hypoglycemia } \\
\hline Symptomatic & 18 & 45 \\
\hline Asymptomatic & 22 & 55 \\
\hline \multicolumn{3}{|l|}{ Symptoms of Hypoglycemia ${ }^{*}$} \\
\hline Jitteriness & 10 & 55.55 \\
\hline Convulsions & 4 & 22.22 \\
\hline Apneic spells & 3 & 16.66 \\
\hline Lethargy & 5 & 27.77 \\
\hline \multicolumn{3}{|l|}{ Maternal risk factors ${ }^{\dagger}$} \\
\hline Diabetes mellitus & 2 & 5 \\
\hline Ante partum hemorrhage & 4 & 10 \\
\hline Pregnancy induced hypertension $(\mathrm{PIH}$ & 13 & 32.5 \\
\hline Twin pregnancy & 4 & 10 \\
\hline \multicolumn{3}{|l|}{ Neonatal risk factors ${ }^{\dagger}$} \\
\hline Birth asphyxia & 6 & 15 \\
\hline $\begin{array}{l}\text { Intrauterine growth restriction } \\
\text { (IUGR) }\end{array}$ & 14 & 35 \\
\hline Prematurity & 23 & 57.5 \\
\hline Sepsis & 3 & 7.5 \\
\hline
\end{tabular}


*Multiple symptoms may be present in a single baby

${ }^{\dagger}$ Multiple Risk factors may be present in a single case

hypoglycemia constituted about $8.26 \%$ of our total Neonatal intensive care unit admissions. Majority (57.5\%) of hypoglycemic babies were preterm babies and $42.5 \%$ were term babies. More number of male babies (57.5\%) had hypoglycemia with male to female ratio of 1.35:1. Asymptomatic hypoglycemia was noticed in more number of babies (55\%) than symptomatic hypoglycemia (45\%). (Table.1)

Asymptomatic hypoglycemia was predominantly noticed in preterm babies $(65.21 \%)$ than that of term babies. Term babies $(58.82 \%)$ showed more symptoms with hypoglycemia than preterm babies. (Figure.1)

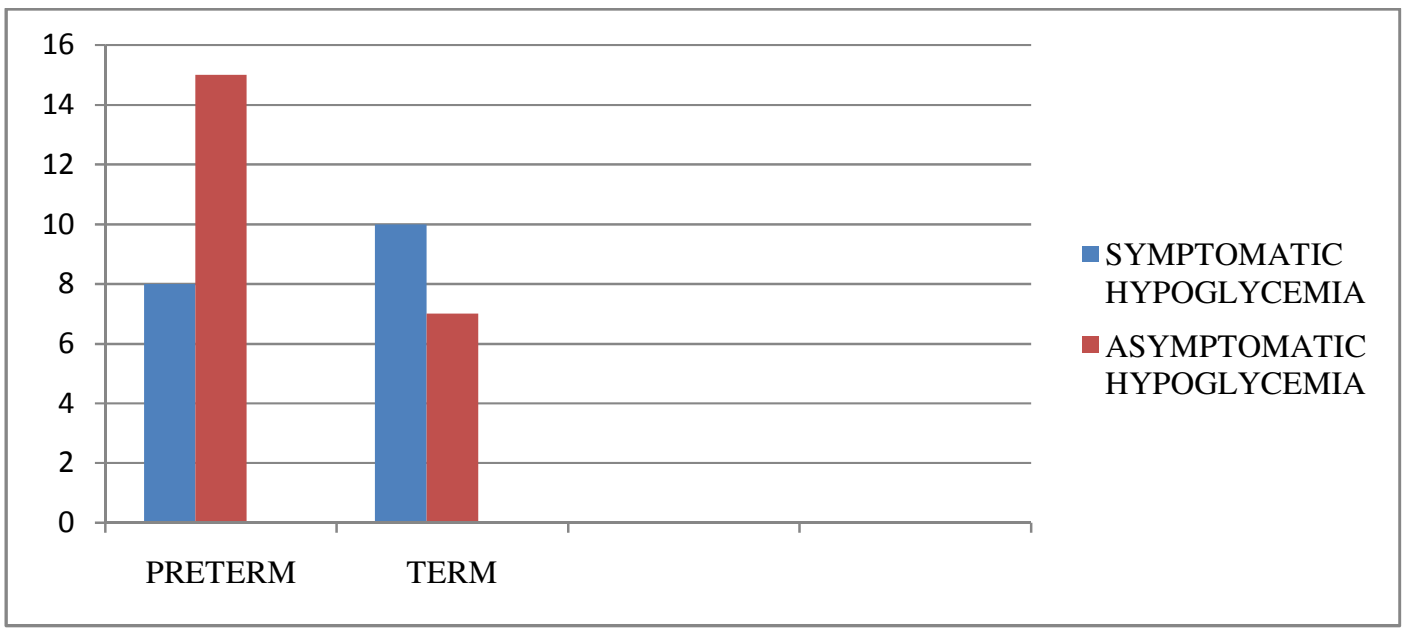

Figure-1: Distribution of cases presenting with and without symptoms according to gestational age

The major clinical manifestations were jitteriness (55. 55\%) followed by lethargy (27.77\%), convulsions (22.22\%) and apneic spells (16.66\%). We noticed PIH (Pregnancy induced hypertension) as the most significant maternal risk factor associated, accounting for $32.5 \%$. We found prematurity and IUGR the most associated neonatal risk factors for neonatal hypoglycaemia. Significantly low Sugar levels (p value $<0.005$ ) were noticed in symptomatic hypoglycemic babies when compared to asymptomatic hypoglycemic babies. (Table.2)

Table 2: Comparison of mean blood sugar levels with standard deviations between symptomatic and asymptomatic cases.

\begin{tabular}{|c|c|}
\hline $\begin{array}{c}\text { Number of Cases } \\
\mathbf{n}=\mathbf{4 0}\end{array}$ & $\begin{array}{c}\text { Mean blood sugar levels (mg/dL) with standard } \\
\text { deviations }\end{array}$ \\
\hline $\begin{array}{c}\text { Symptomatic } \\
(\mathrm{n}=18)\end{array}$ & $26.17^{+} 4.004 \mathrm{mg} / \mathrm{dl}$ \\
\hline $\begin{array}{c}\text { Asymptomatic } \\
(\mathrm{n}=22)\end{array}$ & $30.64^{+} 5.113 \mathrm{mg} / \mathrm{dl}$ \\
\hline
\end{tabular}

*statistically significant with p values $<0.005$ (t value 3.100 and 3.025$)$

\section{Discussion}

Neonatal hypoglycemia is a common metabolic disease due to inability to maintain glucose homeostasis. The overall prevalence depends on definition of hypoglycemia, criteria for diagnosis of hypoglycemia, diagnostic methods and other factors. Hence there is wide range of difference in incidence of hypoglycemia ranging from 4 to $15 \%$. Hypoglycemia can be symptomatic and asymptomatic. Undiagnosed Hypoglycemia can have long term neurological consequences; thus the 
emphasis is on prevention and early detection along with treatment of hypoglycemia. The reported incidence of hypoglycemia varies a lot due to controversies regarding definition of neonatal hypoglycemia and criteria for diagnosis of hypoglycemia. A study from Iran has taken blood sugar levels less than $50 \mathrm{mg} / \mathrm{dl}$ as criteria for diagnosis of hypoglycemia [4]. Similar to some recent Chinese and Indian studies [5, 6]. we have taken blood sugar levels less than $40 \mathrm{mg} / \mathrm{dl}$ as criteria for diagnosis of hypoglycemia [Table 3].

Table-3: Comparison of incidence of hypoglycemia with other studies.

\begin{tabular}{|c|c|c|c|}
\hline Study & $\begin{array}{c}\text { No. of babies admitted } \\
\text { to nicu }\end{array}$ & $\begin{array}{c}\text { No. of babies with } \\
\text { hypoglycemia }\end{array}$ & Incidence \\
\hline Present Study & 484 & 40 & $11.76 \%$ \\
\hline Ghaemi Nosrat et al [5] & 927 & 109 & $10.38 \%$ \\
\hline $\begin{array}{c}\text { Dhananjay CD and } \\
\text { Kiran B et al [6] }\end{array}$ & 366 & 38 & $6.1 \%$ \\
\hline N. Najati et al [4] & 852 & 52 & $6.26 \%$ \\
\hline
\end{tabular}

In our study we found maximum number of preterm babies followed by term babies which is comparable with a Turkish study [7]. In the present study there were no post term deliveries. There was male preponderance with male to female ratio of 1.35:1 which is comparable with the recent Chinese and Indian studies [5,6] with male to female ratio of 1.3:1 and 1.37:1 respectively. Hypoglycemia can present without apparent symptoms, the so called asymptomatic hypoglycemia found in neonates at risk of hypoglycemia. In our study most babies (55\%) had asymptomatic hypoglycemia which is comparable with an Indian study (59.3\%) from Kerala [8]. Neonatal Hypoglycemia can have variable presentation. In our study jitteriness was accounting for $55.55 \%$ of cases where as a western study [9] noticed jitteriness in about $62.7 \%$ of cases. We noticed convulsions in about $22.22 \%$ of cases, where as recent western studies $[9,10]$ noticed convulsions in about $37.5 \%$ and $16.6 \%$ respectively. Apneic spells in the present study $(16.66 \%)$ were comparable with that of a western study $(17.3 \%)[9]$.

Lethargy constituted $27.77 \%$ of cases in our study which is relatively lower than the western study (30\%) [9]. There were various maternal risk factors for neonatal hypoglycemia. In present study we found that Pregnancy induced hypertension (PIH) was the most significant risk factor associated with hypoglycemia, accounting for $32.5 \%$ of cases, followed by Antepartum hemorrhage and twin pregnancy. Out of the all risk factors observed by recent Indian [8] \& western [5] studies it was also seen that PIH was the most significant maternal risk factor associated with hypoglycemia accounting for about $16.5 \%$ and $15.62 \%$ of cases respectively. Out of the neonatal risk factors studied in the present study we found that prematurity $(57.5 \%$ ) was the most significant risk factor followed by Intrauterine growth restriction (IUGR) (35\%), birth asphyxia (15\%), sepsis $(7.5 \%)$.

The risk factors studied by a western study[5] has also shown that prematurity was the most significant risk factor accounting for about $43.1 \%$ of cases, followed by Birth asphyxia, IUGR and Sepsis. One recent Indian study [8] has shown that IUGR was the most significant risk factor accounting for about $29.6 \%$ of cases, followed by prematurity and birth asphyxia.

\section{Conclusions}

Neonatal hypoglycemia constituted about $8.26 \%$ of NICU admissions. Hypoglycemia in neonates can have variable presentations indicating the need for detailed and thorough examination for evidence of hypoglycemia. Identification of risk factors of hypoglycemia and proper monitoring blood glucose levels should be done to plan early treatment and prevent neurological damage.

\section{List of abbreviations Used}

LGA: Large for gestational age, SGA: Small for gestational age, NICU: Neonatal intensive care unit, IUGR: Intrauterine growth restriction, PIH: Pregnancy induced hypertension

Funding: Nil, Conflict of interest: Nil Permission from IRB: Yes 
Original Research Article

\section{References}

1. Jane Hawdon. Metabolic disease: In: Janet M Rennie. Robertson's Textbook of Neonatology. $4^{\text {th }}$ ed. Philadelphia: Elsevier; 2005. p.851-856.

2. Richard E. Wilker, Hypoglycemia and Hyperglycemia In: John. P. Cloherty, Eric C. Eichenwald, Ann R. Stark editors. Manual Of Neonatal Care. $6^{\text {th }}$ Ed. Philadelphia: Wolters Kluwer; 2010. p. 540- 546.

3. Satish C. Kalhan, Prabhu S. Parimi. Metabolic and Endocrine Disorders. In: Richard J. Martin, Avory A Fanaroff, Michele C. Walsh editors. Fanaroff and Martins Textbook, Neonatal- Perinatal Medicine. $8^{\text {th }}$ ed.,vol 2, Mosby Elsevier; 2006. p. 1467-1480.

4. Najati N, Saboktakin L. Prevalence and underlying etiologies of neonatal hypoglycemia. Pak J Biol Sci. 2010 Aug 1;13(15):753-6.

5. Ghaemi Nosrat, Mohammadzadeh Ashraf, Farhat Ahmad Shah. Incidence and etiology of hypoglycemia in neonatal intensive care unit admissions. Journal of Chinese Clinical Medicine. 2009; 4(6): 321-324.
6. Dhananjaya $\mathrm{CD}$, Kiran. B. Clinical profile of hypoglycemia in newborn babies in rural hospital setting. Int J Biol Med Res. 2011; 2(4): 1110 - 1114.

7. Dorina Rodica Burdan, Valentin Botiu, Doina Teodorescu. Neonatal hypoglycemia. The incidence of the risk factors in Salvator Vuia obstetrics gynecology hospital, Arad. TMJ. 2009; 59(1): 77-80.

8. Sasidharan CK, Gokul E, Sabitha S. Incidence and risk factors for neonatal hypoglycaemia in Kerala, India. Ceylon Med J. 2004 Dec;49(4):110-3.

9. Inayatullah Khan, Taj Muhammad, Muhammad Qasim Khan. Frequency and clinical characteristics of Symptomatic Hypoglycemia in neonates. Gomal Journal of Medical Sciences. 2010 Jul; 8(2): 117-120.

10. Nasrin Dashti, Nahid Einollahi, Sakineh Abbasi. Neonatal hypoglycemia: Prevalence and clinical manifestations in Tehran Children's Hospital. Pak J Med Sci. 2007; 23(3):340-343.

\section{How to cite this article?}

Manjunatha Babu R, Joy L. P. D’Souza, Susheela C. Study of incidence, clinical profile and risk factors of neonatal hypoglycemia in a tertiary care hospital. Int. J PediatrRes.2016;3(10):754-758.doi:10.17511/ijpr.2016.i10.07 